Original Research Paper

\title{
Comparative Study of Packet Scheduling Algorithm in LTE Network
}

\author{
Alaa Omer Najim, Nor Asilah Wati Abdul Hamid, \\ Zurina Mohd Hanapi and Idawaty Ahmad \\ Department of Computer Science and Information Technology, \\ Universiti Putra Malaysia, 43400 UPM, Serdang, Malaysia
}

\author{
Article history \\ Received: 06-07-2017 \\ Revised: $17-08-2017$ \\ Accepted: 23-11-2017 \\ Corresponding Author: \\ Nor Asilah Wati Abdul Hamid \\ Faculty of Computer Science \\ and Information Technology, \\ Universtit Putra Malaysia, \\ Malaysia \\ Email: asila@upm.edu.my
}

\begin{abstract}
At present, Long-Term Evolution (LTE) is the most popular Internet access technology because of its robust wireless connectivity that can support data, voice, video and messaging traffic. This advantage has significantly increased the number of users availing themselves of these services through various devices. Such an increase in the number of users has also increased network traffic. Thus, scheduling algorithms are needed to prioritize and distribute available resources among users to improve system performance. Researchers have proposed several algorithms that focus on meeting the Quality of Service requirement for different applications. The current study evaluates the performance of four scheduling algorithms for LTE downlink transmission, namely, proportional fair, exponential PF, exponential rule and log-rule algorithms, in a certain scenario. The performance of these algorithms was evaluated using an LTE-Sim simulator. Simulation results show that the log-rule algorithm outperforms other packet scheduling algorithms in terms of delay, throughput and packet loss ratio when serving Voice-overInternet Protocol packet data service on the LTE network.
\end{abstract}

Keywords: Long-Term Evolution, Cellular Network, Downlink Packet Scheduling, Quality of Service, Delay, Throughput, Packet Loss Ratio, Area Spectral Efficiency

\section{Introduction}

Long-Term Evolution (LTE) mobile broadband networks have attracted considerable attention in research and industrial fields. LTE has been introduced by 3 GPP to satisfy the growing demand for data transmission (Ferdosian et al., 2016). As a step toward the fourth generation of mobile technology, LTE can ensure improved user experience by providing multimedia and Internet applications even in highmobility situations. Furthermore, common network performance indices, such as throughput, low delay and complex traffic models, are usually considered in LTE unlike in other access technologies.

Radio Resource Management (RRM) is a challenging LTE issue that is rated using the aforementioned network performance indices. Pavithira and Prabakaran (2016) studied RRM, which involves scheduling mechanisms and algorithms used to increase network efficiency. Orthogonal frequency division multiple access and evolved NodeB (eNB) constitute the scheduling mechanism for Downlink (DL) channels. eNB is a base station that is intended to deliver a high data rate with low latency values for various users who are multiplexed in time and frequency domains ( $\mathrm{Li}$ et al., 2016). Thus, resource scheduling at eNB must be conducted with utmost care to achieve the required performance and a high satisfaction level of the end users.

Decisions on the scheduling process are finalized at the MAC layer of the LTE protocol stack. These decisions become critical and difficult to arrive at especially when prioritizing mixed user flows in a single network traffic scenario. Technically, this condition implies that various user applications have different characteristics and therefore require different specifications of Quality of Service (QoS) features to be met. For example, Real-Time (RT) traffic applications supplied by the base station have different QoS requirements as each application requests for a specific network feature (e.g., low delay, minimal Packet Loss Ratio (PLR), high amount of throughput and area 
spectral efficiency (Makki et al., 2017)) that needs to be fulfilled to guarantee the efficiency of the application on the network to meet LTE QoS requirements defined by Ferdosian et al. (2016).

The current study aims to analyze several scheduling algorithms used commonly for RT traffic such as Voice over Internet Protocol (VoIP) and video in a specific system model. Such a system model restricts all users from being connected to the base station at the cell edge area. With this assumption, we aim to test the behavior of resource distribution over flows of different types applied by various well-known scheduling algorithms. Notably, existing studies have not considered such a scenario in the performance evaluation of cell-edge users. The scheduling algorithms simulated include Proportional Fair (PF), Exponential PF (EXP/PF), exponential rule (EXP-Rule) and log-rule. Scheduling is one of the main features of LTE systems because of its main task of distributing available resources among active users to satisfy their QoS requirements.

This paper is organized as follows: Section 2 describes the DL system scheduling model in LTE and related studies that focus on common scheduling schemes considered in this study. Section 3 presents the complexity analysis. Section 4 discusses the experimental evaluation and simulation parameters. Section 5 reports the results of the scheduling algorithms. Section 6 concludes the paper.

\section{System Scheduling in LTE}

This section presents a background on the system model of the LTE packet scheduler and describes four schemes of resource allocation in DL LTE systems.

\section{System Model}

In LTE network, DL packet scheduling involves several processes that are repeatedly triggered in every Time Transmission Interval (TTI). This process also involves both UE and eNB. The process is initiated when UE measures its Channel Quality Indicator (CQI) and sends it to eNB via Uplink (UL) channel. Figure 1 illustrates a simplified model of radio resource scheduling in the DL LTE system (Capozzi et al., 2013). CQI and QoS parameters from RLC and application layers are then used by the eNB for allocation decisions and Resource Block (RB) distribution among flows. Time Domain (TD) and Frequency Domain (FD) can be used when selecting the suitable Modulation and Coding Scheme (MCS) in the physical layer to achieve high performance and ensure minimal block errors. Finally, results of scheduling decisions and the session information are sent back to UE through a packet data control channel, which engages in a proper packet data shared channel payload if it is scheduled.

Resource scheduling at the MAC layer is iterated at three TTIs; the scheduler is assumed to know the channel gain for each sub-carrier and each user. However, due to limited signaling channel resources, sub-carriers are grouped into RBs, each consisting of 12 adjacent subcarriers, as shown in Fig. 2 (Tran et al., 2012). Each RB has a duration of $0.5 \mathrm{~ms}$, which corresponds to 6 or 7 OFDM symbols depending on whether a normal or extended cyclic prefix is used. The smallest resource unit that can be allocated to a user is a physical RB, which consists of two consecutive RBs that span a sub-frame duration or TTI of $1 \mathrm{~ms}$ and a bandwidth of $180 \mathrm{kHz}$.

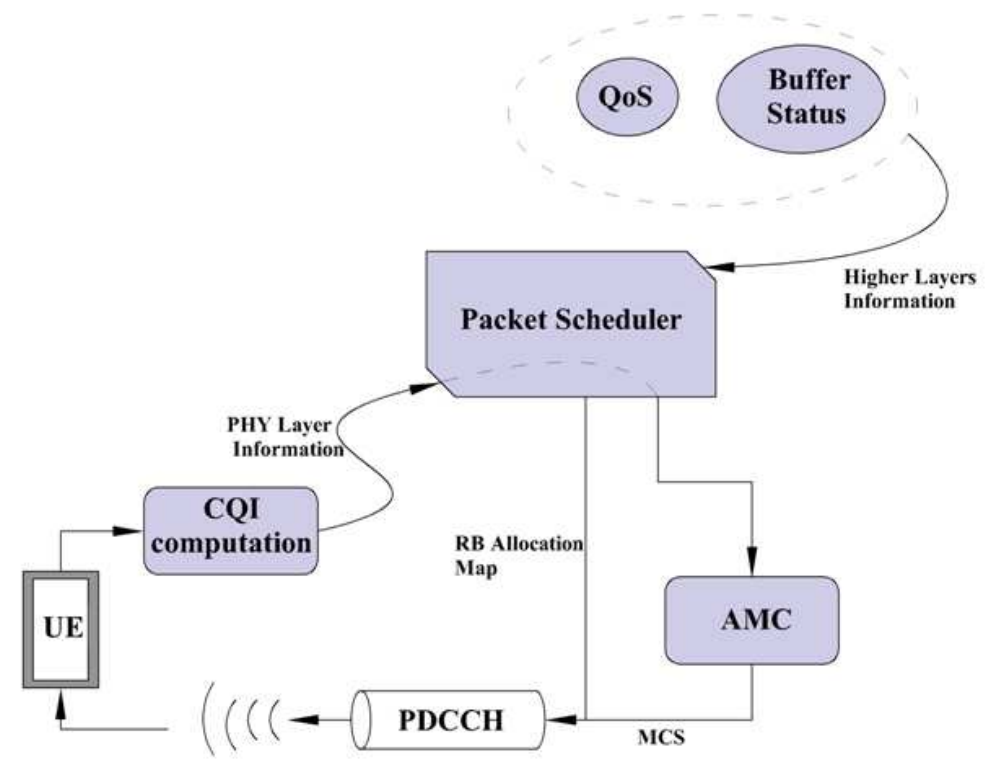

Fig. 1: Basic model of downlink packet scheduling process in LTE 


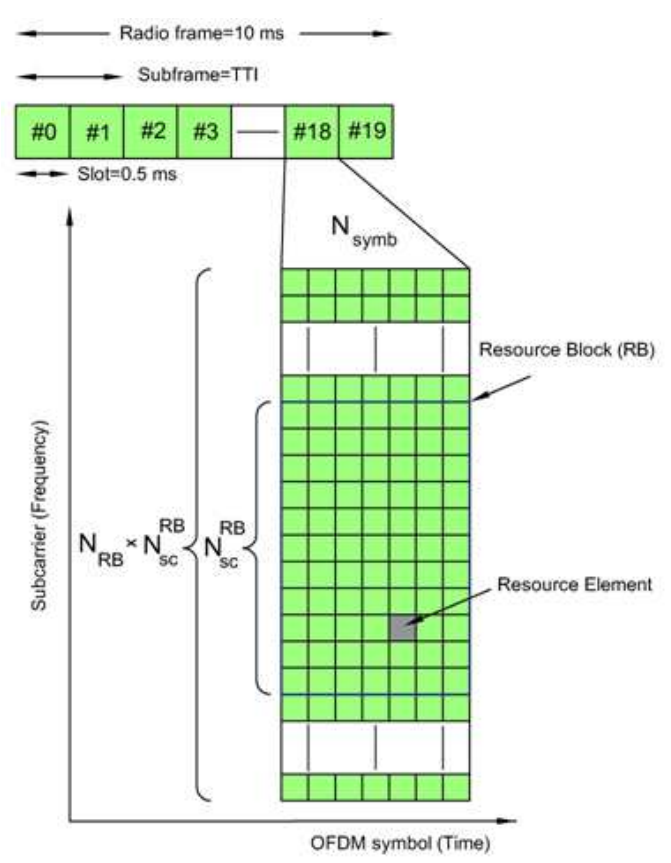

Fig. 2: LTE frame structure in TD/FD

The process can be divided into a sequence of the scheduler, which calculates a metric for each stream that can be allocated.

\section{Related Studies}

Numerous studies on LTE scheduling have been conducted due to the importance of the scheduler in determining the overall system performance. Basukala et al. (2009; Alfayly et al., 2012) studied the performance of two well-known packet scheduling algorithms, namely, $\mathrm{EXP} / \mathrm{PF}$ and M-LWDF; one of these algorithms was developed for single-carrier wireless systems that supporti multimedia services with video streaming and web browsing features. Several other authors focused on VoIP applications in terms of Quality of Experience and other QoS metrics (e.g., PLR and delay). Nsiri et al. (2015) proposed a scheduler that was more complex than the PF algorithm to increase the overall system throughput while maintaining a fair approach. Several other schemes achieve satisfactory scheduling performance in providing multimedia services Liu and Chen (2015). Ali et al. (2016) also proposed an allocation scheme with excellent performance in packet loss rate and delay because it exploits deadline properties.

In the following sections, four scheduling algorithms, namely, PF, EXP-PF, EXP-Rule and logrule algorithms, are used in simulation scenarios. To satisfy the QoS requirements of video users, the scheduler that aims to guarantee bounded delay will the suitable scheme because one of the main requirements of RT flows is to deliver the data packet within a certain period (Liu et al., 2013).

\section{PF Scheduler}

This scheduling algorithm is a classical fairness standard that maintains a tradeoff between maximum rate and fairness (Kelly, 1997). The fairness concept in $\mathrm{PF}$ is derived from Round Robin fairness principle. Thus, it attempts to schedule UEs with good CQIs while preventing other users from starving by imposing a fairness level of rate distributions. The scheduler assigns RBs to flows with the highest priority. This priority is obtained from the following metric:

$$
\begin{aligned}
& x_{i, n}^{P F}=\frac{d_{i, n}(t)}{R_{i}(t-1)} \\
& R_{i}(t-1)=(1-\beta) r_{i}(t)+\beta R_{i}(t-1) \forall 0 \leq \beta \leq 1 \\
& T_{f}=\frac{1}{(1-\beta)}
\end{aligned}
$$

where, $d_{i, n}(t)$ is the estimated data rate for the $i$-th flow at time $t$ on the $n$-th RB and $R_{i}(t)$ is the previous average data rate when the $i$-th user flow is at time $t$. As shown in Equation (1), the PF mechanism depends on previous average throughput, which acts as a weighting factor of the incoming data rate. Therefore, users with low CQIs can still be served within a certain amount of time. The parameter $\beta$ in Equation (2) plays an important role because it influences the value of time window $T_{f}$ in Equation (3), where fairness should be imposed according to the relationship. 


\section{EXP/PF Scheduler}

In scenarios where delay-sensitive traffic exists, PF starts treating all flows similarly, thereby reducing the performance of RT traffic. Thus, Rhee et al. (2003) proposed a modified PF principle that can achieve flexible behavior with RT/NRT traffic. EXP/PF is an integration of PF and a developed EXP term. EXP properties guarantee the throughput for RT services and the PF properties maintain a minimum level of service for NRT to ensure fairness in services. The $\mathrm{EXP} / \mathrm{PF}$ metric strictly depends on the service type prioritized by the RT metric.

$$
x_{i, n}^{E X P / P F}=\exp \left(\frac{\alpha_{i} q_{i}-\omega}{1+\sqrt{\omega} a}\right) \cdot \frac{d_{i, n}(t)}{R_{i}(t-1)}
$$

where, $\omega$ and $\alpha_{i}$ are given from:

$$
\begin{aligned}
& \omega=\frac{1}{N_{R T}} \sum_{i=1}^{N_{R T}} \alpha_{i} q_{i} \\
& \alpha_{i}=\frac{-\log \delta_{i}}{\tau_{i}}
\end{aligned}
$$

where, $q_{i}$ is the queue size for the $i$-th flow. $\omega$ is the weight factor of the exponential term that enables maximization of throughput while maintaining fairness, because it accounts for the average values of $q_{i} . \alpha_{i}$ is a system parameter obtained from the acceptable PLR for the $i$-th user, $\delta_{i}$, over the maximum delay bound $\tau_{i}$. $\alpha$ represents the flows with high requirements in terms of accepted data rate. The metric for the NRT is the typical $\mathrm{PF}$ rule in Equation (1). The preceding discussion demonstrates that EXP/PF attempts to consider RT flows by strictly maintaining fairness when imposing the PF term and recording average values of queue sizes, which attempts to reach high amounts of throughput by prioritizing flows with large queue sizes (Ang et al., 2015). Services with NRT fairness and minimum data rate can be achieved by applying PF.

\section{EXP-Rule Scheduler}

Shakkottai and Stolyar (2002) proposed and defined EXP-Rule as a throughput-optimal scheduling scheme for high data-rate networks. Thus, this algorithm has been extensively used to develop more complex scheduling decisions. EXP-Rule is considered an enhanced version of the EXP/PF scheme because it highly prioritizes flows on the basis of their achieved throughput. EXP-Rule is a service-based priority metric. For RT traffic applications, this algorithm uses the following formula:

$$
x_{i, n}^{\text {EXP-Rule }}=b_{i} \exp \left(\frac{a_{i} q_{i}}{c+\sqrt{\left(1 / N_{R T}\right) \Sigma_{j} q_{i}}}\right) \cdot \varphi
$$

where, $\varphi$ is the spectral efficiency of the $i$-th UE on the $n$-th RB. $a, b$ and $c$ are the optimality parameters that, according to Sadiq et al. (2009), should be set to $\left[5 /\left(0.99 \tau_{i}\right), 10 /\left(0.99 \tau_{i}\right)\right], 1 / \mathrm{E}\left[\varphi_{i}\right]$ and 1 , respectively. EXP-Rule attempts to equalize the weighted queue size of all the flow queues to ensure that if a queue is larger than others, then the EXP term becomes larger, which in turn enables the flow to be prioritized on the basis of the archived system throughput. By contrast, a flow with a smaller queue size than others results in a small EXP term value that is close to 1 ; in this case, the rule behavior is toward maintaining fairness for the flow. For NRT flows, the PF metric is used.

\section{Log-Rule Scheduler}

The log-rule algorithm is an opportunistic delaybased scheme proposed by Sadiq et al. (2011). In contrast with the EXP-rule algorithm, the log-rule algorithm prioritizes flows by disregarding the balance of unequal queue sizes in favor of building a tradeoff between low delay amount and good level of throughput. In the scheduling metric of log-rule algorithm to prioritize RT services, optimality parameters $a, b$ and $c$ have the same values as those of the EXP-Rule algorithm:

$x_{i, n}^{\text {LOG-Rule }}=b_{i} \log \left(c+a_{i} q_{i}\right) \cdot \varphi_{i, n}$

where, optimality parameters $a, b$ and $c$ have the same values as in EXP-Rule.

\section{Complexity Analysis}

The complexity analyses of PF, EXP-PF, EXP-Rule and log-rule algorithms are based on their allocation time. Although PF has to select among $n$ users, its scheduler allocation complexity is equal to $\mathrm{O}(\log n)$. EXP-PF algorithm includes a weighting parameter to the $\mathrm{PF}$ metric. Its computational time differs from that of PF, but it also has to select among $n$ users. Thus, its allocation complexity is $\mathrm{O}(\log n)$. Similarly, the EXP rule and log-rule algorithm compute the exponential and logarithmic value of a delay parameter to calculate the priority metric. Thus, these algorithms have an allocation complexity of $\mathrm{O}(\log n)$.

To finalize our discussion and help the reader, all pros and cons of the QoS-aware strategies discussed, as well as parameters they use for computing scheduling metrics and the complexity analysis, have been summarized in Table 1.

\section{Simulation and Performance Evaluation}

A well-known simulation tool called LTE-Sim simulator (Piro et al., 2011) that encompasses various 
aspects of an LTE network was used to evaluate the performance of the scheduling algorithms (PF, EXPPF, EXP-Rule and log-rule). This section describes an LTE scenario and how the output results are managed. The performance evaluation of the LTE-Sim simulator is then presented.

\section{LTE Topology Building}

Using an LTE-Sim simulator, an LTE scenario can be created as a static function in $\mathrm{C}++$ header file. The reference of this function should be added to the main program. The following basic scenario can be created directly from the main menu:

- Create an instance for Simulator, NetworkManager, FlowsManager and FrameManager components

- $\quad$ Create Cell, ENodeB and $U E$ objects using methods of the NetworkManager class. For each of these objects, several parameters can be assigned directly with the constructor of the class

- Create applications and define each of their source and destination, QoS parameters, IP classifier parameters, start time and stop time

- Define the duration of the simulation and, finally, call the Simulator::Run() function

To simplify the use of the simulator, a single-cell LTE topology is proposed as an example that is stored in the Simulation/Scenarios folder, where the number of UEs and the number of flows can be specified by command line.

\section{Tracing Results}

The current version of the simulator provides a stateof-the-art tracing feature. The trace is displayed directly during the simulation. An example of the output trace is reported. In the figure, the first field describes the event that has triggered the tracing. In particular, rows that start with TX, RX and DROP are associated with packets that have been sent, received and dropped, respectively. The second field describes the packet type in which the trace referring to other fields are described, as follows:

- Identifier uniquely determines the packet

- Bearer ID identifies the bearer used to map the packet

- Source ID identifies the node that sends the packet

- Destination ID identifies the node that receives the packet

- Time represents the instant in which the packet is created

- Delay represents the delay of the received packet

\section{Simulation Parameters}

An LTE network composed of 19 cells and with a radius equal to $1 \mathrm{~km}$ was simulated. Simulation was performed using $\mathrm{C}++$ and Shell programming. The simulation was run on Fedora Linux Core 19, an operating system that runs on a Linux Core i5 processor machine with a $3.2 \mathrm{GHz} \mathrm{CPU}$ and $8 \mathrm{~GB}$ of RAM.

Table 1: Comparison analysis of downlink scheduling algorithms

\begin{tabular}{|c|c|c|c|c|c|}
\hline Author/year & $\begin{array}{l}\text { Scheduling } \\
\text { scheme }\end{array}$ & $\begin{array}{l}\text { Parameters used } \\
\text { by each scheduler }\end{array}$ & Pros & Cons & Complexity \\
\hline (Kelly, 1997) & $\mathrm{PF}$ & $\begin{array}{l}\text { - SINR } \\
\text { - Throughput }\end{array}$ & $\begin{array}{l}\text { Maintain trade-off } \\
\text { between throughput } \\
\text { and fairness }\end{array}$ & $\begin{array}{l}\text { It is not a good } \\
\text { choice for RT } \\
\text { applications }\end{array}$ & $\begin{array}{l}\log \\
\text { complexity }\end{array}$ \\
\hline $\begin{array}{l}\text { (Rhee, Holtzman } \\
\text { et al., 2003) }\end{array}$ & EXP-PF & $\begin{array}{l}\text { - SINR } \\
\text { - Throughput } \\
\text { - HoL delay packet } \\
\text { - Target delay } \\
\text { - Target PLR }\end{array}$ & $\begin{array}{l}\text { support RT services } \\
\text { with different QoS } \\
\text { requirements and } \\
\text { NRT data services }\end{array}$ & $\begin{array}{l}\text { Offers higher } \\
\text { priority to the RT } \\
\text { service users } \\
\text { over NRT service } \\
\text { users if their HOL } \\
\text { delays are } \\
\text { approaching delay } \\
\text { deadline }\end{array}$ & $\begin{array}{l}\log \\
\text { complexity }\end{array}$ \\
\hline $\begin{array}{l}\text { (Shakkottai and } \\
\text { Stolyar, 2002) }\end{array}$ & EXP-Rule & $\begin{array}{l}\text { - SINR } \\
\text { - Throughput } \\
\text { - HoL delay packet } \\
\text { - Target delay }\end{array}$ & $\begin{array}{l}\text { Throughput oriented } \\
\text { scheduling }\end{array}$ & $\begin{array}{l}\text { High delay especially } \\
\text { on small traffic }\end{array}$ & $\begin{array}{l}\log \\
\text { complexity }\end{array}$ \\
\hline $\begin{array}{l}\text { (Sadiq, Baek } \\
\text { et al., 2011) }\end{array}$ & LOG-Rule & $\begin{array}{l}\text { - SINR } \\
\text { - Throughput } \\
\text { - HoL delay packet } \\
\text { - Target delay }\end{array}$ & $\begin{array}{l}\text { Balance between } \\
\text { queues }\end{array}$ & $\begin{array}{l}\text { In burst traffic, } \\
\text { throughput is bad }\end{array}$ & $\begin{array}{l}\log \\
\text { complexity }\end{array}$ \\
\hline
\end{tabular}


Table 2: Simulation parameters

\begin{tabular}{ll}
\hline Parameters & Assumption \\
\hline Simulation duration & $100 \mathrm{~s}$ \\
Carrier frequency & $1.9 \mathrm{GHz}$ \\
Bandwidth & $10 \mathrm{MHz}$ \\
Number of Ues & $20,40,60,80,100$ \\
UE speed & $30 \mathrm{~km} / \mathrm{h}$ \\
eNB transmission radius & $1 \mathrm{~km}$ \\
Number of Cells and eNB & single cell with 1 eNB \\
Transmission power at eNB & $43 \mathrm{dBm}$ \\
Path loss & $28.6+35 \mathrm{log}$ \\
Thermal Noise density & $-174 \mathrm{dBm} / \mathrm{Hz}$ \\
Noise Figure & $7 \mathrm{~dB}$ \\
Max Re-transmission number & 5 \\
\hline
\end{tabular}

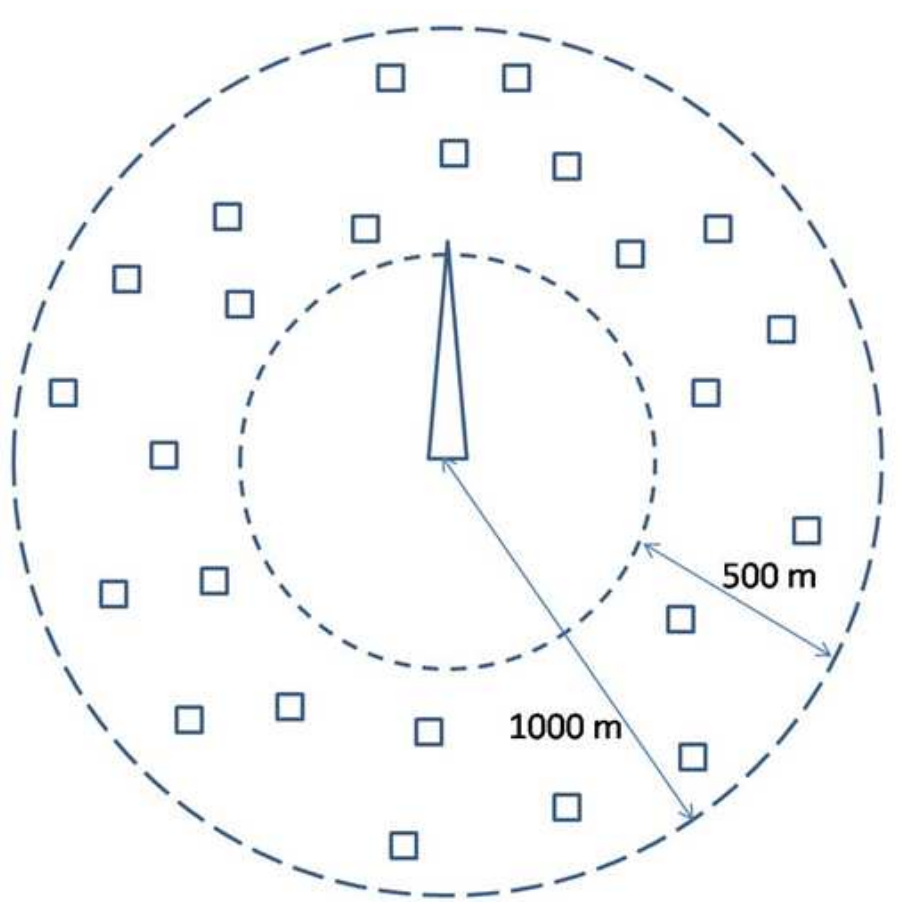

Fig. 3: System model of the simulation scenario (Liu et al., 2015)

The results are depicted using Microsoft Excel Chart Utility version 2013 and LibreOffice Calc version 5. In FD, a bandwidth of $10 \mathrm{MHz}$ is selected for the DL channel with $50 \mathrm{RBs}$ in each TTI. In TD, resource allocation occurs every TTI (TTI $=1 \mathrm{~ms}$ ). The UE number in a cell is set to several cases from 20 to 100 and the initial distance from UE to eNB is uniformly distributed between 500 and $1000 \mathrm{~m}$ (Fig. 3), with a UE speed of $30 \mathrm{~km} / \mathrm{h}$. Traffic sources used were RT (i.e., video and VoIP). Table 2 describes the rest of the parameters implemented within the scenario.

\section{Performance Evaluation}

The performance evaluation of the implemented DL scheduling algorithms (i.e., PF, EXP-PF, EXP-Rule and log-rule algorithms) is reported. The following realistic scenario is considered: 19 cells exist with the radius equal to $1 \mathrm{~km}$, where several UEs in the range [20-100] are uniformly distributed into each cell. UEs travel in the area following the random walk mobility model in an urban macro-cell scenario (Camp et al., 2002).

The entire bandwidth is distributed among four cells in a cluster to guarantee $10 \mathrm{MHz}$ of bandwidth in the DL for each cell. The focus of this study is on RT applications, which includes video and VoIP traffic transmission. Each user receives a H.264 video flow, which is encoded at $128 \mathrm{kbps}$ and one VoIP flow. The performance of any network was measured by the QoS functions experienced by the network user. In our experiments, the system throughput, delay, PLR and area spectral efficiency were used to assess the performance of each algorithm. 
Delay is defined as the latency observed in a transmission packet of a flow from the source to the destination. For video flows, delay is usually $300 \mathrm{~ms}$; for VoIP flows, the delay is $150 \mathrm{~ms}$. Based on the findings, Fig. 4 shows that as the number of video flows increases in the system, delay increases. This increase becomes more visible as the number of users exceeds 40 . PF shows the least increase in delay because it does not count delay. Instead, it emphasizes providing a tight throughput to fairness balancing. The other three algorithms seem to be more suitable for RT videos. The EXP-rule scheduling decisions favor traffic with burst characteristics. This condition contradicts the log-rule principle, which highlights a small traffic, such as VoIP flows. Thus, the delay increase is limited by the effect of the log term. The EXP-PF algorithm plots the most reasonable delay behavior that balances the services among different flow types as the delay term attempts to provide different flows with closed delay weights to ensure that the EXP term has the greatest effect on the scheduling decision.

Throughput represents the successfully transmitted bits through a network. As shown in Fig. 5, the aggregated throughput of different traffic types is illustrated over the increased UE sets. For RT video streaming, packets are transmitted in bursts. Thus, a scheduler, such as EXP-rule algorithm, that considers the actual queue size in such a way that makes the scheduler prioritize flows on the basis of the high value of queue size (e.g., video) exhibits the highest throughput trend.

On VoIP flows, the log-rule algorithm outperforms the three other algorithms; thus, its scheduling decision favors the allocation of more channel resources to the small flows to be transmitted. The EXP-PF algorithm seems to compromise the VoIP throughput for the sake of having a low delay value. The EXP-Rule is considered to have an optimal throughput. This condition means that the scheduling decision increases the throughput at the expense of delay.

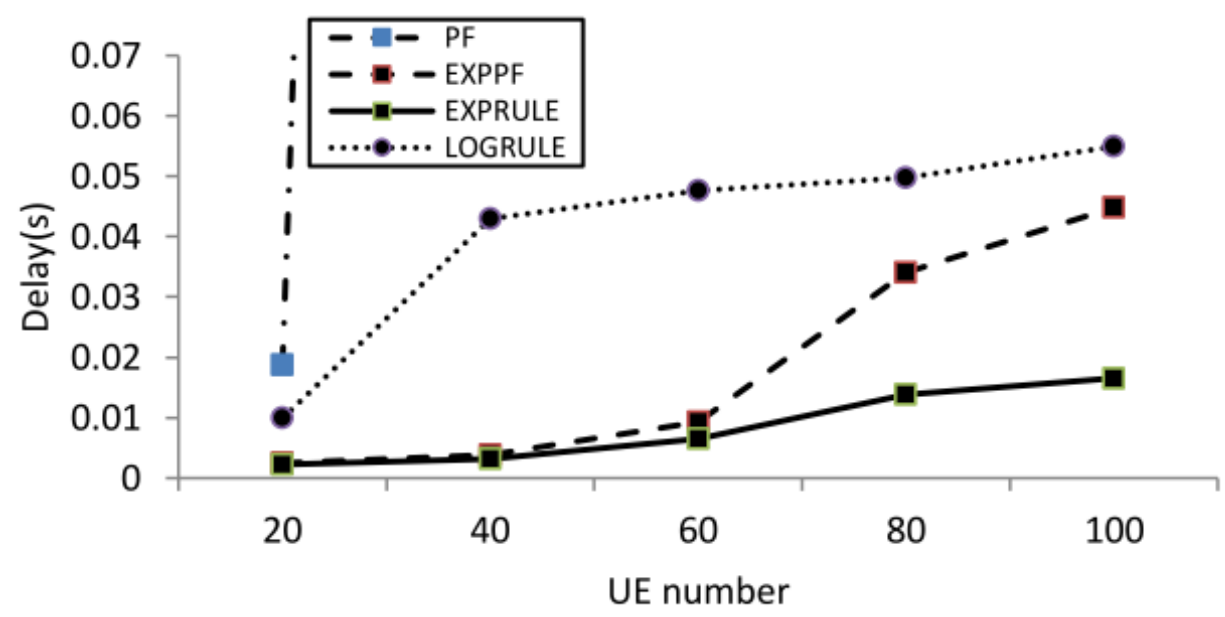

(a)

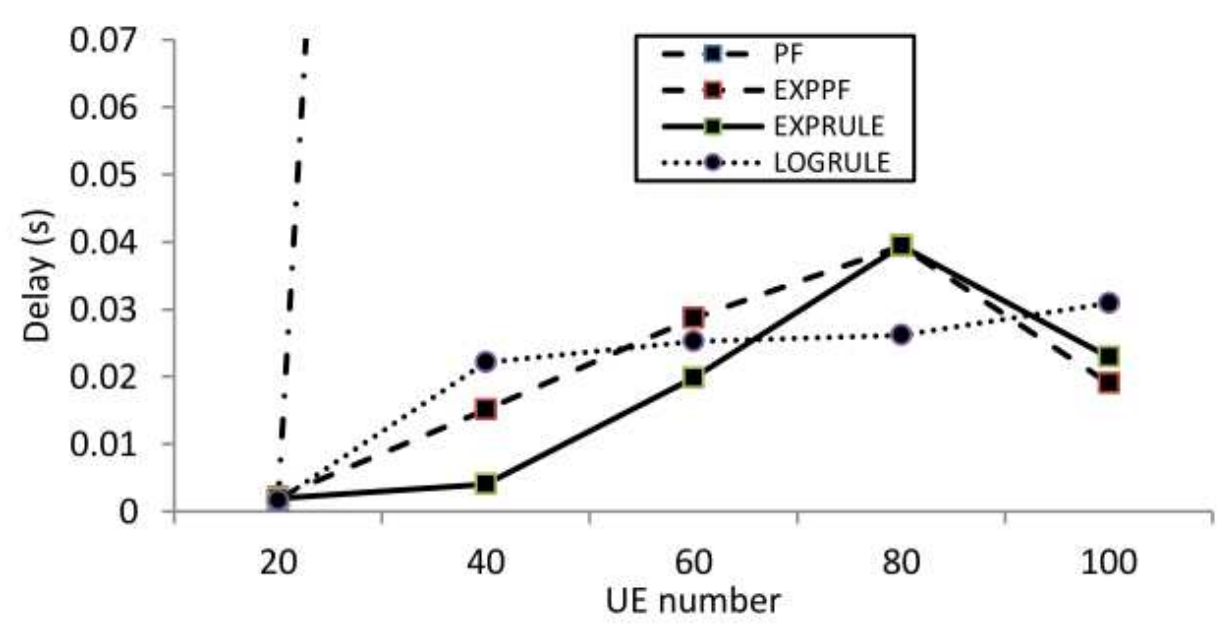

(b)

Fig. 4: Delay for (a) Video and (b) VoIP traffic types 


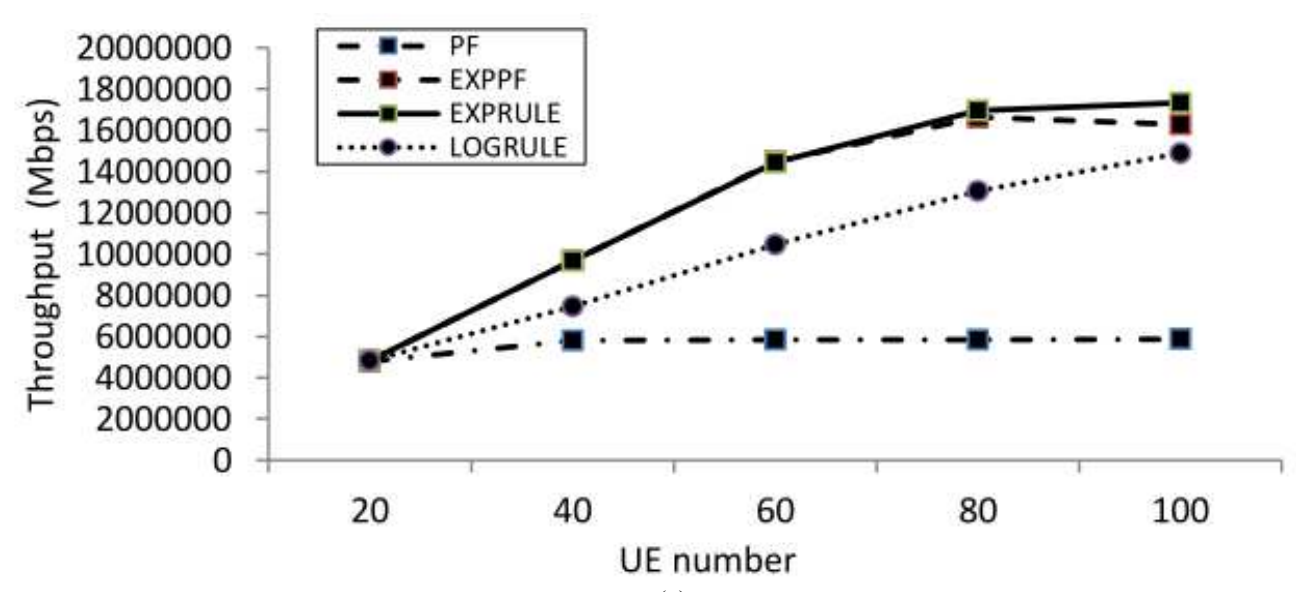

(a)

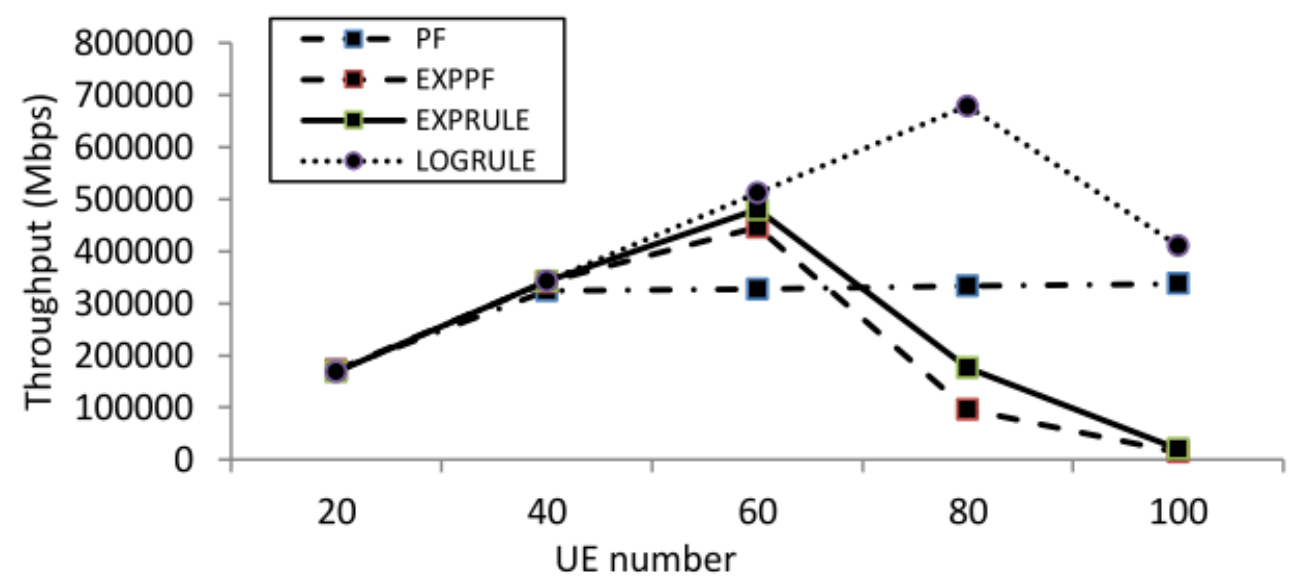

(b)

Fig. 5: System throughput (a) Video (b) VoIP traffic types

PLR represents the ratio of the number of packets of a flow received at the destination to the number of packets transmitted. Its value is usually $10^{-3}$ for VoIP and $10^{-6}$ for video. As shown in Fig. 6, the PLR of video flows is always below $10 \%$ for all simulated scheduling algorithms when the cell is charged among 30 users. When the UE number exceeds 40, the PLRs in the case of PF and log-rule algorithms increase rapidly, whereas the PLRs for EP-PF and EXP-rule algorithms have relatively slow growth. The PLRs in the case of PF and log-rule algorithms are always low. PLR is significantly related to the amount of transmitted bits on each flow. Therefore, the high amount of throughput obtained by log-rule algorithm in the case of VoIP flows indicates that less data are lost in each scheduling interval. This finding explains why several algorithms such as PF have a low throughput. Data loss can be partly attributed to the lifetime of the expired flows in the system or frequent retransmissions; this condition frequently occurs in the case of burst traffic.
Figure 7 demonstrates that area spectral efficiency decreases with the increase in service loads in the cell and when the service load reaches a certain threshold, cell spectral efficiency tends to stabilize. The scheduling algorithm performs in the same manner when the area spectral efficiency increases slightly as in the case of the EXP-Rule algorithm. This finding implies that throughput-based algorithms such as the EXP-Rule algorithm usually favor flows that belong to UEs with high channel quality; therefore, a high MCS order is selected for that flow to use the largest portion of channel resources in data transmission. This condition certainly improves the efficiency of radio channel performance. By contrast, schedulers that consider other QoS characteristics of traffic may have a slight compromise in resource distribution efficiency. Therefore, in this case, the decision is not only intended to obtain the optimal channel quality as much as to fulfill other metrics such as delay, as observed in the case of the log-rule and EXP-PF algorithms. 


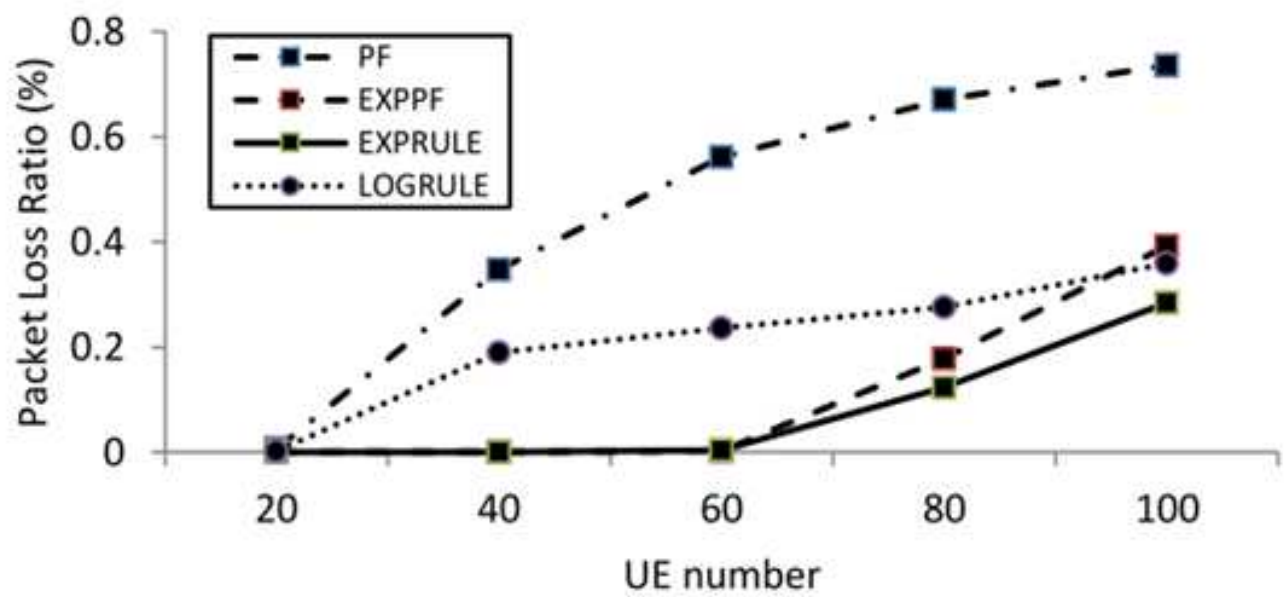

(a)

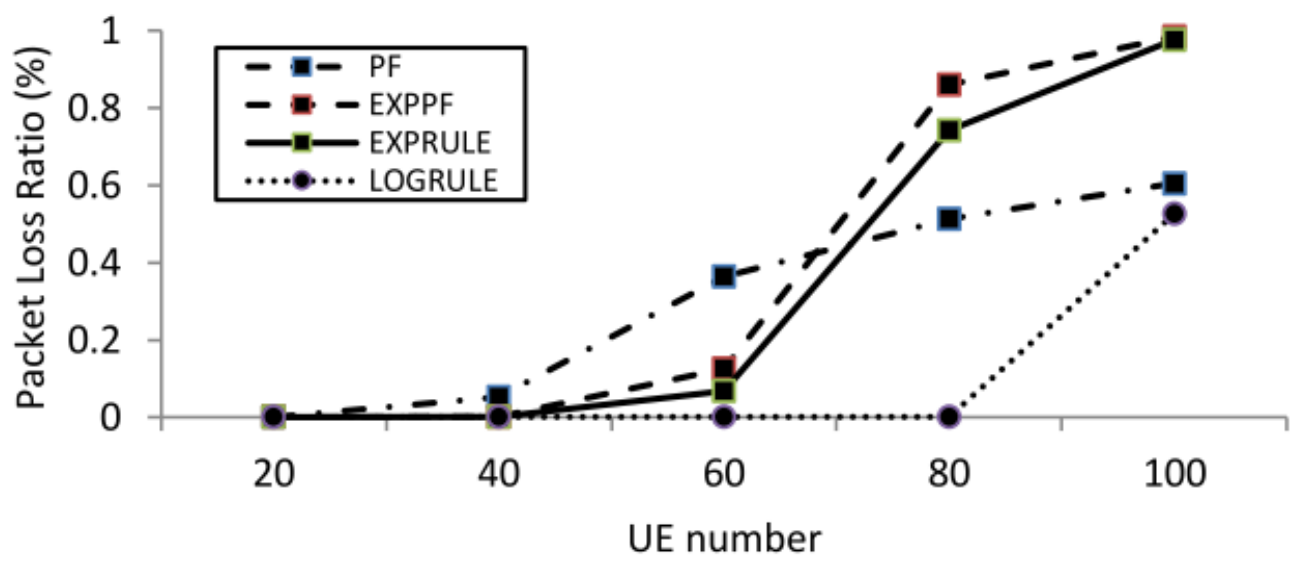

(b)

Fig. 6: PLR for (a) Video and (b) VoIP traffic types

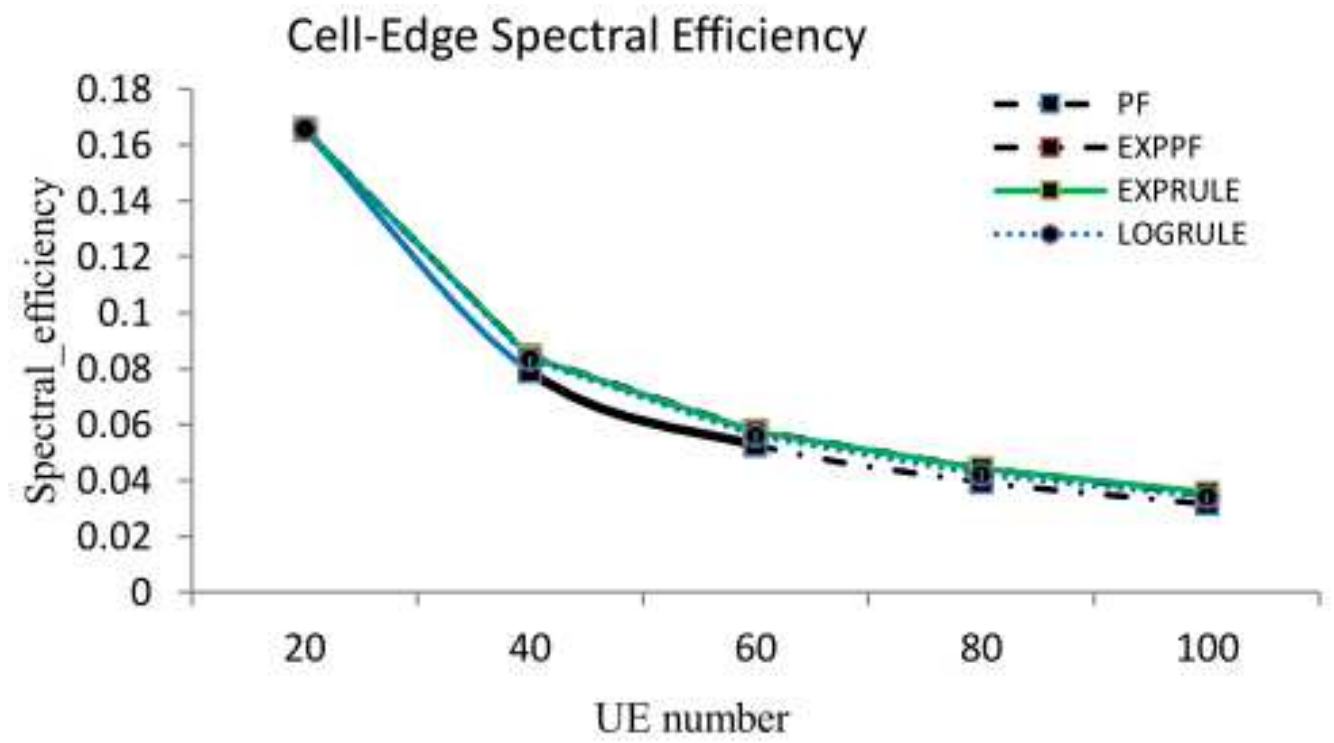

Fig. 7: Area spectrum efficiency 


\section{Conclusion}

This study evaluated the performance of four scheduling algorithms, namely, PF, EXP-PF, EXPRule and log-rule algorithms. These scheduling schemes were studied on the basis of the principle that a good scheduling scheme should improve system performance. In a simulation experiment scenario, the schedulers were evaluated in terms of several metrics, namely, delay, system throughput, PLR and area spectral efficiency. These metrics were adopted to accurately evaluate the performance of the scheduling algorithms. Simulation results show that the log-rule algorithm outperforms the three other packet scheduling algorithms in terms of delay, throughput and PLR when providing VoIP packet data service on an LTE network.

Other complex scheduling problems and the UL and DL directions using femtocell scenario will be investigated in future studies.

\section{Acknowledgement}

This project is sponsored by Fundamental Research Grant Scheme (FRGS), Universiti Putra Malaysia (UPM). Project Code: 08-01-15-1722FR.

\section{Author's Contributions}

Alaa Omer Najim: Conceived of the presented idea, developed the theory and performed the computations as well as carried out the experiment and wrote the manuscript with support from other authors.

Nor Asilah Wati Abdul Hamid: Conceived of the presented idea and verified the analytical methods.

Idawaty Ahmad and Zurina Mohd Hanapi: Verified the analytical methods. All authors discussed the results and contributed to the final manuscript.

\section{Ethics}

There are no ethical issues or conflicts of interest that may arise.

\section{References}

Alfayly, A., I.H., L. Sun and E. Ifeachor, 2012. QoEbased performance evaluation of scheduling algorithms over LTE. Proceedings of the Globecom Workshops, Dec. 3-7, IEEE Xplore Press, Anaheim. DOI: 10.1109/GLOCOMW.2012.6477781

Ali, A.A., R. Nordin, M. Ismail and H. Abdullah, 2016. An efficient scheduling scheme for ofdma resource blocks with joint user scheduling based on earliest deadline first with Carrier Aggregation (CA) in LTE-A system. Wireless Personal Commun., 88: 173-183. DOI: $10.1007 / \mathrm{s} 11277-015-3085-8$
Ang, E.M., K. Wee, Y.H. Pang and K.K. Phang, 2015. A performance analysis on packet scheduling schemes based on an exponential rule for real-time traffic in LTE. EURASIP J. Wireless Commun. Netw., 2015: 1-12. DOI: 10.1186/s13638-015-0429-8

Basukala, R., H.M. Ramli and K. Sandrasegaran, 2009. Performance analysis of EXP/PF and M-LWDF in downlink 3GPP LTE system. Proceedings of the 1st Asian Himalayas International Conference on Internet, Nov. 3-5, IEEE Xplore Press, Kathmandu. DOI: 10.1109/AHICI.2009.5340336

Camp, T., J. Boleng and V. Davies, 2002. A survey of mobility models for ad hoc network research. Wireless Commun. Mobile Comput., 2: 483-502. DOI: $10.1002 / \mathrm{wcm} .72$

Capozzi, F., G. Piro, L. A. Grieco, G. Boggia and P. Camarda, 2013. Downlink packet scheduling in LTE cellular networks: Key design issues and a survey. IEEE Commun. Surveys Tutorials, 15: 678-700. DOI: 10.1109/SURV.2012.060912.00100

Ferdosian, N., M. Othman, B.M. Ali and K.Y. Lun, 2016. Greedy-knapsack algorithm for optimal downlink resource allocation in LTE networks. Wireless Netw., 22: 1427-1440. DOI: $10.1007 / \mathrm{s} 11276-015-1042-9$

Kelly, F., 1997. Charging and rate control for elastic traffic. Eur. Trans. Telecommun., 8: 33-37. DOI: $10.1002 /$ ett.4460080106

Li, Y.P., B.J. Hu, H. Zhu, Z.H. Wei and W. Gao, 2016. A delay priority scheduling algorithm for downlink real-time traffic in LTE networks. Proceedings of the Networking, Electronic and Automation Control Conference on Information Technology, May 20-22, IEEE Xplore Press, Chongqing. DOI: 10.1109/ITNEC.2016.7560452

Liu, B., H. Tian, L. Xu, 2013. An efficient downlink packet scheduling algorithm for real time traffics in LTE systems. Proceedings of the Consumer Communications and Networking Conference, (CNC'13), IEEE Xplore Press, pp: 364-369.

Liu, Q. and C.W. Chen, 2015. Smart downlink scheduling for multimedia streaming over LTE networks with hard handoff. IEEE Trans. Circuits Syst. Video Technol., 25: 1815-1829.

DOI: 10.1109/TCSVT.2015.2400751

Liu, S., C. Zhang, Y. Zhou and Y. Zhang, 2015. Delaybased weighted proportional fair algorithm for LTE downlink packet scheduling. Wireless Personal Commun., 82: 1955-1965. DOI: $10.1007 / \mathrm{s} 11277-015-2324-3$

Makki, B., C. Fang, T. Svensson, M. Nasiri-Kenari and M. Zorzi, 2017. Delay-sensitive area spectral efficiency: A performance metric for delayconstrained green networks. IEEE Trans. Commun., 65: 2467-2480.

DOI: 10.1109/TCOMM.2017.2686395 
Nsiri, B., N. Mallouki, S. Mhatli, M. Ghanbarisabagh and M. Ammar et al., 2015. Modeling and performance evaluation of novel scheduling algorithm for downlink LTE cellular network. Wireless Personal Commun., 83: 2303-2316. DOI: $10.1007 / \mathrm{s} 11277-015-2522-\mathrm{Z}$

Pavithira, S. and N. Prabakaran, 2016. Downlink packet scheduling mechanism in long term evolution technology. Proceedings of the International Conference on Circuit, Power and Computing Technologies, Mar. 18-19, IEEE Xplore Press, Nagercoil, India. DOI: 10.1109/ICCPCT.2016.7530309

Piro, G., L.A. Grieco, G. Boggia, F. Capozzi and P. Camarda, 2011. Simulating LTE cellular systems: An open-source framework. IEEE Trans. Vehicular Technol., 60: 498-513. DOI: $10.1109 /$ TVT.2010.2091660

Rhee, J.H., J.M. Holtzman and D.K. Kim, 2003. Scheduling of real/non-real time services: Adaptive EXP/PF algorithm. Proceedings of the 57th Semiannual Vehicular Technology Conference, Apr. 22-25, IEEE Xplore Press, Jeju, South Korea.

DOI: 10.1109/VETECS.2003.1207583
Sadiq, B., R. Madan and A. Sampath, 2009. Downlink scheduling for multiclass traffic in LTE. EURASIP J. Wireless Commun. Netw., 2009: 1-18. DOI: $10.1155 / 2009 / 510617$

Sadiq, B., S. J. Baek and G. De Veciana, 2011. Delayoptimal opportunistic scheduling and approximations: The log rule. IEEE/ACM Trans. Netw., 19: 405-418. DOI: 10.1109/TNET.2010.2068308

Shakkottai, S. and A.L. Stolyar, 2002. Scheduling for multiple flows sharing a time-varying channel: The exponential rule. Translations Am. Math. SocietySeries, 2: 185-202. DOI: 10.1090/trans2/207/12

Tran, T.T., Y. Shin and O.S. Shin, 2012. Overview of enabling technologies for 3GPP LTE-advanced. EURASIP J. Wireless Commun. Netw., 2012: 1-12. 